\title{
Single-Stage Photodiode OP-Amp Solution Suited for a Self-Mixing FMCW System
}

\author{
Daniel Nordin and Kalevi Hyyppä
}

\begin{abstract}
The current delivered by the photodiode in a self-mixing frequency modulated continuous wave or optical frequency domain reflectometry system consists of a dc-current resulting from the local oscillator, the reflected signal, dark current in the photodiode, and current generated from background light. The current also contains the useful harmonic signal with a beat frequency corresponding to the range and radial velocity of a target. To avoid saturation and clipping due to the dc current generated in the photodiode, it is desirable to minimize the gain at dc while maintaining a high gain in the beat frequency region. We have investigated some different solutions and present a modified current-to-voltage converter using bootstrapping and added voltage gain, which addresses this problem using only one OP-amp and no de shorting inductors.
\end{abstract}

Index Terms-Frequency modulated continuous wave (FMCW), operation amplifier, optical frequency domain reflectometry (OFDR), photodiode, transimpedance amplifier.

\section{INTRODUCTION}

F REQUENCY MODULATED CONTINUOUS WAVE (FMCW) is a range and radial velocity measuring technique well-known from the field of radar applications. The reflected part of an outgoing frequency modulated wave (w1) is mixed with a local oscillator wave (w2), and as a result the frequency difference between the two signals can be obtained. If the frequency modulation is chosen as a triangular wave, both radial velocity and range of the target can be determined from the frequency difference, usually referred to as beat frequency. By using a tunable laser as the source, with the light frequency as the carrier frequency and a photodiode as detector, the beat frequency can be extracted directly from the photodiode current. This phenomena, usually referred to as the "self-mixing-effect," is due to the fact that the photodiode delivers a current proportional to the squared sum of the two electrical fields $w 1$ and w2. The most common type of lasers used are diode lasers of DFB or DBR type, $\mathrm{CO}_{2}$ or Nd:YAG [1]-[14]. Systems can be built either as classical open Michelson interferometers or with optical fibers. Optical frequency domain reflectometry (OFDR) is closely related to FMCW. Both utilize the same principles, but in the case of OFDR the technique is used to study optical fiber networks.

Besides the beat frequency, a dc current will also be generated in the photodiode. This paper deals with different ways, while focusing on one of them, to avoid saturation due to this dc current and still maintain a high gain in the beat frequency region.

Manuscript received September 12, 2001; revised August 11, 2003.

The authors are with the Division of Industrial Electronics, Lulea University of Technology, Lulea, Sweden.

Digital Object Identifier 10.1109/TIM.2003.820454

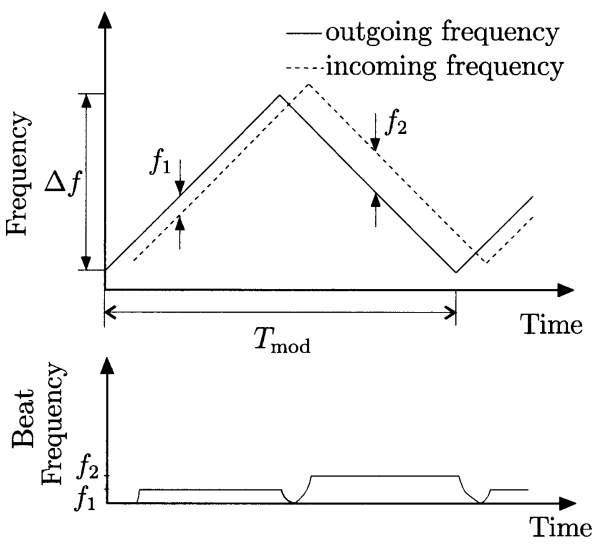

Fig. 1. Triangular modulation scheme where the outgoing frequency corresponds to the local oscillator wave and the incoming frequency corresponds to the reflected wave. The lower part shows the corresponding beat frequencies.

Before going into the problem description and the solution we start with a short introduction to the theory behind FMCW.

\section{FMCW THEORY}

\section{A. Frequency Modulation}

If triangular modulation of the optical frequency is used, as displayed in Fig. 1, the range $R_{a}$ can be calculated from the frequency shift $f_{R}$ corresponding to the distance according to

$$
f_{R}=\frac{f_{1}+f_{2}}{2}=\frac{4 R_{a} \cdot \Delta f}{c \cdot T_{\text {mod }}}
$$

where $c$ is the speed of light, $f_{1}$ and $f_{2}$ the output beat frequencies as shown in Fig. 1, and $T_{\text {mod }}$ the modulation cycle. The target velocity $v_{t}$ can be calculated from the Doppler shift $f_{D}$ according to [15]

$$
f_{D}=\frac{f_{2}-f_{1}}{2}=\frac{2 \cdot v_{t}}{\lambda}
$$

where a positive $v_{t}$, and hence $f_{D}$, corresponds to a decreasing distance between source and target. The above calculations are derived under the assumptions that the range and speed of the target are constant during one modulation period and that $\left|f_{D}\right|<f_{R}$.

\section{B. Self-Mixing Effect}

If the received electrical field is denoted as $E_{r} \cos \left(\omega_{r} t+\theta_{r}\right)$, and the local oscillator electrical field is denoted $E_{l o} \cos \left(\omega_{l o} t+\right.$ $\left.\theta_{l o}\right)$, the optical power received by the photodiode, ignoring reflections at the photodiode, will equal

$$
\Phi(t)=\frac{A}{Z}\left(E_{r} \cos \left(\omega_{r} t+\theta_{r}\right)+E_{l o} \cos \left(\omega_{l o} t+\theta_{l o}\right)\right)^{2}
$$


where $A$ is the active area of the detector and $Z$ the characteristic impedance of the medium in front of the detector. By expanding (3) and removing terms with frequencies too high for the photodiode to respond to, the current from the diode can be expressed as

$$
\begin{aligned}
i(t)= & \Re \Phi(t)+I_{B} \\
= & \frac{\Re A}{Z}\left(\frac{E_{l o}^{2}}{2}+\frac{E_{r}^{2}}{2}+E_{l o} E_{r}\right. \\
& \left.\quad \times \cos \left(\omega_{b} t+\theta_{r}-\theta_{l o}\right)\right)+I_{B}
\end{aligned}
$$

where $f=2 \pi \omega_{b}$ is the beat frequency, $\Re$ the responsivity of the detector and $I_{B}$ the dc current in the detector, which can include both dark current and current generated by background light. The total dc current $I_{D}$ generated by the photodiode can hence be expressed as

$$
I_{D}=\frac{\Re A}{Z}\left(\frac{E_{l o}^{2}}{2}+\frac{E_{r}^{2}}{2}\right)+I_{B} .
$$

It should be pointed out that (4) is only valid when the two waves have the exact same polarization states and that polarization mismatch degrades the performance.

\section{PROBLEM DESCRIPTION}

In a system where most of the transmitted power will be lost, it can be shown theoretically that maximum signal to noise ratio for the photodiode is obtained by transmitting most of the available power [16]. But even for such a design the dc current, resulting mainly from the local oscillator, can cause saturation or clipping in the amplifier if no special care is taken to avoid it.

When combining an OP-amp with a photodiode for current to voltage conversion it is often advantageous to keep the voltage over the photodiode constant. Commonly a constant reverse bias or zero voltage is desired. The latter case can be accomplished by placing the photodiode between the OP-amps (+) and (-) inputs and utilize feedback. One possible solution to avoid saturation due to the dc current is to place a high pass filter between the photodiode and the first amplifying stage. A drawback with this approach is that it forces you to use a reverse biasing source and the resulting reverse bias over the diode will vary due to variations in the dc current. A second drawback is that the filter introduces noise.

Another possibility is to use a cascade of two OP-amps. The gain in the first stage must be kept low, to avoid saturation and clipping, and a high pass filter that removes the dc level is inserted between the stages. The disadvantages of this approach are the extra OP required and the noise that it will contribute directly in the signal path. Yet another possibility is to utilize inductances as a de short circuit. However, when a wide passband, starting from a few kilohertz, is desired the resulting inductance value tend to be of the order of $\mathrm{mH}$ or larger, and hence unpractically large.

\section{SOLUTION}

Our circuit is based on a current to voltage converter with added voltage gain using bootstrapping [17], as displayed in Fig. 2. The current is first converted to voltage by $R_{1}$. The voltage obtained over $R_{1}$ is then amplified by the voltage gain $\left(1+R_{3} / R_{2}\right)$. The voltage over the photodiode is kept constant

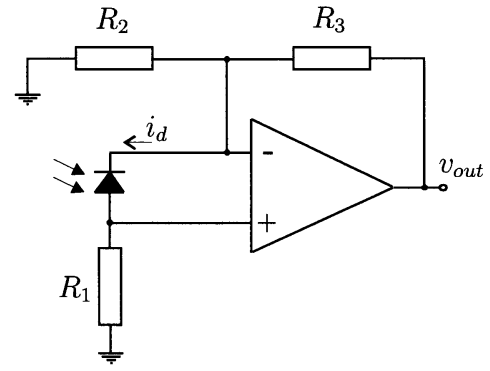

Fig. 2. A current to voltage converter using bootstrapping and added voltage gain.

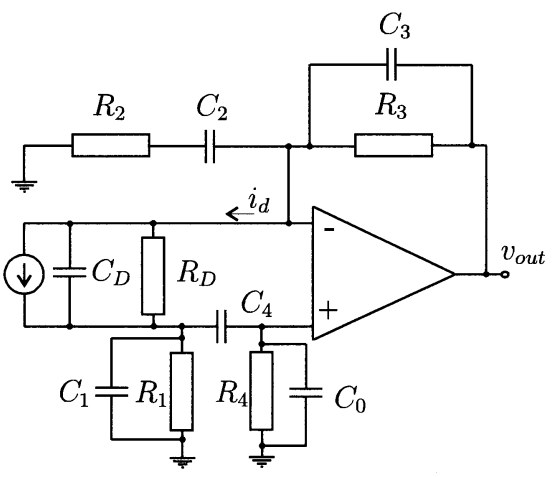

Fig. 3. Our modified circuit with minimal gain at dc. The photodiode has been replaced with an equivalent model.

by placing it between the OP-amp inputs, refereed to as bootstrapping. By including $C_{2}$, as seen in Fig. 3, the gain at lower frequencies and up to the passband can be expressed as

$$
\frac{V_{\text {out }}}{I_{d}}=R_{3}+R_{1}\left(1+\frac{j \omega R_{3} C_{2}}{j \omega R_{2} C_{2}+1}\right) .
$$

When deriving (6) we have assumed infinite open loop OP-amp gain. From now on, this assumption is always made when deriving transfer functions.

Adding only $C_{2}$ to the circuit in Fig. 2 reduces the gain at dc by reducing the voltage gain $1+R_{3} / R_{2}$ to unity. The total gain at dc then equals $R_{1}+R_{3}$, since the dc current generated in the photodiode will propagate through both of these resistances. In Fig. 3 the photodiode has also been replaced with an equivalent model consisting of a current source, shunt capacitor $C_{D}$ and a leakage resistance $R_{D}$. Note that a complete equivalent model should also include an ideal diode in parallel with the current source. $R_{D}$ is usually larger than $10^{8} \Omega$ and can often be approximated as infinite. If the dc gain must be reduced even further, compared to (6), we can insert $C_{4}$ and $R_{4}$. The gain for the circuit at lower frequencies and up to the passband then equals

$$
\frac{V_{\text {out }}}{I_{d}}=R_{3}+\frac{j \omega R_{1} R_{4} C_{4}}{j \omega C_{4}\left(R_{1}+R_{4}\right)+1}\left(1+\frac{j \omega R_{3} C_{2}}{j \omega R_{2} C_{2}+1}\right) .
$$

Inserting $C_{4}$ grounds the OP-amp (+) input at dc, creating a virtual ground at the (-) input that reduces the dc gain for the entire circuit to simply $R_{3}$. However, this introduces the same drawbacks as placing a high pass filter between the photodiode and the first amplifier. Capacitors $C_{0}, C_{1}$ and $C_{3}$ influence stability, bandwidth and shunts noise. The complete transfer function with all capacitors included can be obtained from (7) and is 


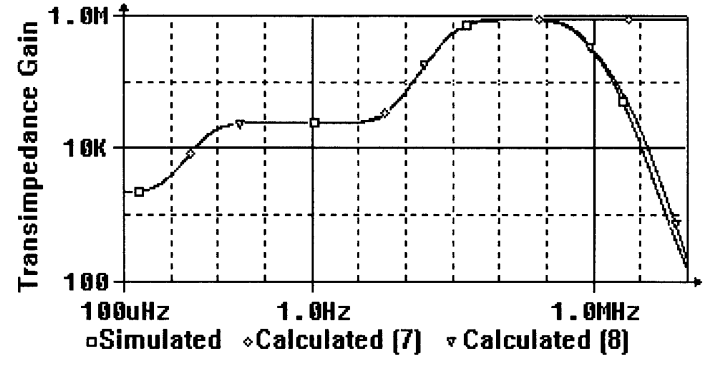

Fig. 4. SPICE simulated transfer function and the calculated ones from (7) and (8).

expressed in (8), where $C_{d}=C_{0}+C_{i c m}$, as the common mode input capacitance of the OP $C_{i c m}$ appears in parallel with $C_{0}$ [see at (8) at the bottom of page]. Fig. 4 displays the simulated transfer function and the ones obtained from (7) and (8). As (8) assumes infinite open loop gain, a slight difference between the calculated and the simulated gain can be observed at higher frequencies. OP-amp OPA655 and the component values in Table I were used in the simulation. The listed values of $C_{i c m}$ and the differential input capacitance of the OP $C_{i d m}$, used in the calculation, were obtained from the data sheet for the OPA655. The value of $R_{3}$ was selected based on tests done in our FMCW system.

With $C_{4}$ and $R_{4}$ inserted reverse bias should be added to avoid forward biasing of the photodiode. Reverse biasing can be accomplished by inserting a voltage source between $R_{1}$ and ground. One drawback associated with the bootstrapping placement is that the voltage obtained over $R_{4}$, and hence over both of the OP-amp inputs, is a common mode signal that will be amplified by the none-zero common mode gain of the OP-amp resulting in an unwanted contribution to $v_{\text {out }}$. It is also possible to remove the voltage gain by shorting the output to the (-) input. Doing so, in conjunction with inserting $C_{4}$ and $R_{4}$, completely removes the gain at dc but also decreases the overall gain.

\section{A. Noise Performance}

A complete noise analysis including all noise sources, as seen in Fig. 5, and transfer functions in the circuit is tedious due to the large number of components and the circuit layout. We have therefore chosen to focus on the main contributors separately.

The photodiode generates a noise current consisting of both thermal and shot noise. Usually the shot noise resulting from the dc current through the diode junction will be the dominant one. The noise current generated in the photodiode will be added to the useful signal current and both will be amplified by the circuits transfer function. It is hence not interesting from a circuit analysis point of view.
TABLE I

PARAMETERS USED IN THE SPICE SimULATIONS AND CALCULATIONS

\begin{tabular}{c|c|c}
\hline$R_{1}=22 \mathrm{k} \Omega$ & $R_{2}=56 \Omega$ & $R_{3}=2.2 \mathrm{k} \Omega$ \\
\hline$R_{4}=10 \mathrm{M} \Omega$ & $R_{D}=1 \mathrm{G} \Omega$ & - \\
\hline$C_{1}=10 \mathrm{pF}$ & $C_{2}=2.2 \mu \mathrm{F}$ & $C_{3}=10 \mathrm{pF}$ \\
\hline$C_{4}=2.2 \mu \mathrm{F}$ & $C_{0}=10 \mathrm{pF}$ & $C_{D}=3 \mathrm{pF}$ \\
\hline$C_{i c m}=1.0 \mathrm{pF}$ & $C_{i d}=1.2 \mathrm{pF}$ & - \\
\hline
\end{tabular}

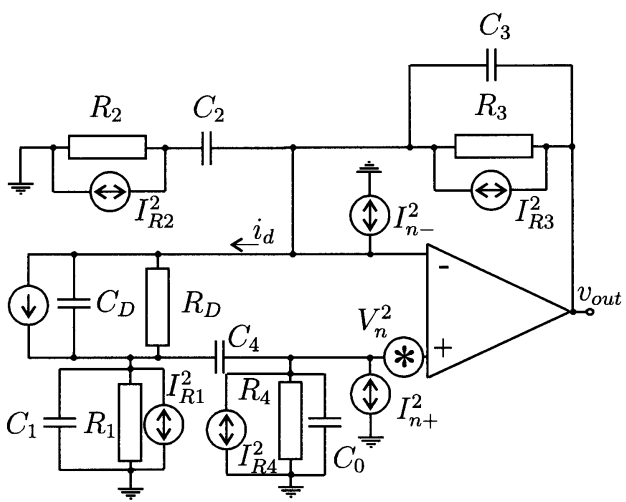

Fig. 5. Noise model of our circuit without the noise sources associated with the photodiode.

The gain for the root spectral density of the OP-amp input noise voltage $V_{n}$, neglecting $C_{4}$, assuming $R_{1} \gg R_{2}$ and infinite $R_{D}$, can be expressed as

$$
\begin{aligned}
\frac{V_{n o}}{V_{n}}=\mid \begin{array}{l}
1+\frac{j \omega R_{3} C_{2}}{\left(j \omega C_{2} R_{2}+1\right)\left(j \omega C_{3} R_{3}+1\right)} \mid \\
\cdot\left|\frac{1+j \omega R_{x}\left(C_{D}+C_{i d}+C_{0}+C_{1}+C_{i c m}\right)}{1+j \omega R_{x}\left(C_{0}+C_{1}+C_{i c m}\right)}\right|
\end{array}
\end{aligned}
$$

where $R_{x}=R_{1} / / R_{4}$. A SPICE simulation of the input voltage noise gain and the calculated one from (9) are displayed in Fig. 6.

The input voltage noise gain gives rise to noise gain peaking at higher frequencies. The voltage gain $\left(1+Z_{3} / Z_{2}\right)$ itself gives rise to one peak and the second factor in (9) gives rise to a second peak. As the second factor will be multiplied by one as $Z_{3} / Z_{2} \rightarrow 0$, the second peak will not be completely cut off until limited by the decreasing gain of the OP-amp at higher frequencies. The difference between the simulated and calculated curves are assumed to result from our assumption of infinite OP-amp gain at all frequencies. We also used a slightly larger $C_{D}$ than commonly found in photodiodes for these types of applications, to visualize the second peak around $1 \mathrm{MHz}$.

$$
\begin{aligned}
\frac{V_{\text {out }}=}{I_{d}} & \frac{R_{3}}{j \omega C_{3} R_{3}+1} \\
& +\frac{j \omega R_{1} R_{4} C_{4}}{j \omega\left(C_{4} R_{1}+C_{4} R_{4}+C_{d} R_{4}+C_{1} R_{1}\right)-\omega^{2}\left(C_{4} C_{d} R_{1} R_{4}+C_{4} R_{4} C_{1} R_{1}+C_{d} R_{4} C_{1} R_{1}\right)+1} \\
& \cdot\left(1+\frac{j \omega R_{3} C_{2}}{\left(j \omega C_{3} R_{3}+1\right)\left(j \omega R_{2} C_{2}+1\right)}\right)
\end{aligned}
$$




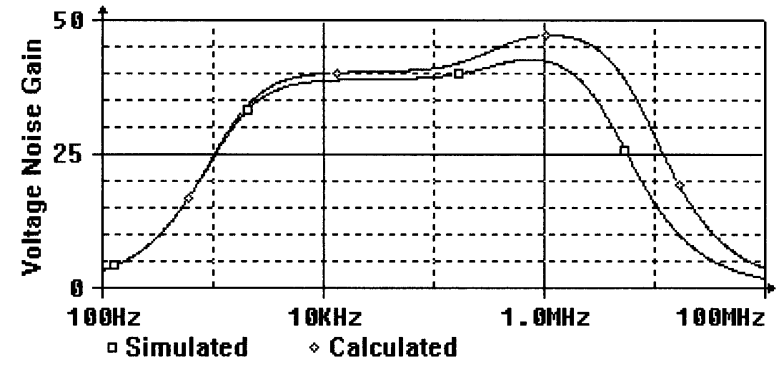

Fig. 6. SPICE simulated and from (9) calculated noise voltage gains.

The transfer function of the root spectral density of the input current noise $I_{n+}$ to the output can be expressed as

$$
\frac{V_{n o}}{I_{n+}}=\left|\frac{R_{x}}{j \omega R_{x} C_{x}+1}\right| \cdot\left|1+\frac{j \omega C_{2} R_{3}}{\left(j \omega R_{2} C_{2}+1\right)\left(j \omega R_{3} C_{3}+1\right)}\right|
$$

where $C_{x}=C_{1}+C_{0}+C_{i c m}$. The simulated and calculated current noise gains are displayed in Fig. 7. As $I_{n-}$ only propagates trough $R_{3} / / C_{3}$ without being amplified by the voltage gain its contribution will be significantly lower than that of $I_{n+}$.

Thermal noise contributions in the circuit can be kept low by using relatively small resistance values. However, this may not always result in the largest signal to noise ration. The parallel combination of $R_{4}$ and $R_{1}$ will usually be the largest thermal noise contributor as the noise current $I_{n R x}=\sqrt{4 k T / R_{x}}$ appears in parallel with $I_{n+}$ and will hence be amplified with the same gain function.

\section{B. Choice of Components}

Minimal gain at dc, without including any inductors, is achieved by minimizing $R_{3}$, and $R_{1}$ if $C_{4}$ is not used. By keeping both $R_{1}, R_{3}$, and $R_{2}$ relatively low, but $R_{3} \gg R_{2}$, the dc gain can be kept low while the voltage gain still make a significant contribution to the overall gain. $R_{2}$ and $R_{3}$, $C_{2}$ would also have to be chosen to obtain the desired low frequency break points. The break point due to the zero equals $\left(2 \pi R_{3} C_{2}\right)^{-1}$ and the one due to the pole $\left(2 \pi R_{2} C_{2}\right)^{-1}$. If $R_{1} \ll R_{4}, R_{x} \rightarrow R_{1}$ will determine the overall gain in the passband. $R_{x}$ also effects both the signal gain, as seen in (7), and the noise. Increasing $R_{x}$ increases the current noise gain as seen in (10), with the same amount as the useful signal gain. The thermal noise contribution from $R_{x}$ at the output increases with $\sqrt{R_{x}}$, as $R_{x}$ increases. Increasing $R_{x}$ also shifts the beginning of the noise gain peaking, due to the input voltage noise, to lower frequencies. If the input voltage noise peaking effect dominates the total noise spectra a larger value of $R_{x}$ and $R_{3}$ can improve the overall signal to noise ratio. Break points due to the capacitors $C_{0}, C_{1}$ and $C_{3}$ can be approximated as

$$
f_{C 1}=\frac{1}{2 \pi R_{x} C_{1}}, f_{C 0}=\frac{1}{2 \pi R_{x}\left(C_{0}+C_{i c m}\right)}
$$

and

$$
f_{C 3}=\frac{1}{2 \pi C_{3} R_{3}}
$$

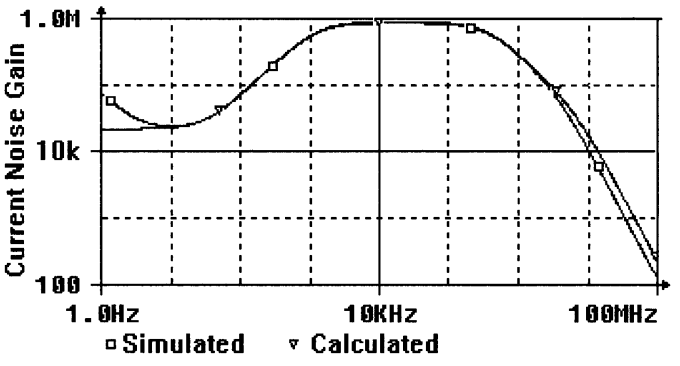

Fig. 7. SPICE simulated and from (10) calculated current noise gains.

\section{COMPARISONS}

The output noise of a regular current to voltage converter, also refereed to as a transimpedance amplifier, consist of contributions from three different noise sources. First, the input voltage noise of the OP-amp will result in a noise gain peaking at higher frequencies according to

$$
\frac{V_{n o}}{V_{n}}=\left|\frac{1+j \omega R_{f}\left(C_{D}+C_{i d m}+C_{i c m}+C_{f}\right)}{1+j \omega R_{f} C_{f}}\right|
$$

where $R_{f}$ and $C_{f}$ are the feedback resistance and capacitance. Second, the input current noise of the OP-amp will propagate through $R_{f} / / C_{f}$ generating a noise voltage at the output. Third, $R_{f}$ itself will also contribute thermal noise. Increasing $R_{f}$ increases the gain but also amplifies the input current noise by the same amount and increases the self generated thermal noise at the output by $\sqrt{R_{f}}$. The beginning of the noise gain peak due to the input voltage noise is also shifted to lower frequencies as $R_{f}$ increases. If the noise contribution from the input voltage noise dominates the noise spectra, which is common, increasing $R_{f}$ can improve the overall signal-to-noise ratio. However, the high gain at dc excludes a transimpedance circuit with very large gain as a practical solution in our case, unless one introduces a high pass filter after the photodiode, but before the op-amp. Then it is still possible to use this circuit and the drawbacks with this solution were discussed in Section III.

If a cascade of two amplifier stages are used the dc level can be completely removed before the second stage by inserting a simple high pass link after the first stage. The first stage in the cascade usually consists of a current to voltage converter and the second stage commonly consisting of a none-inverting voltage amplifier. The gain in the first stage can be kept as high as possible without risking saturation and cutting of the useful signal. The noise generated by all three noise sources in the first stage, as mentioned above, will be amplified by the voltage gain of the second stage along with the input voltage noise of the second amplifier. In this case, the input voltage noise of the second amplifier does not generate any noise gain peaking at the output and the other noise sources generated by the second stage can often be neglected in comparison.

Compared to our circuit, the cascade has similar or slightly higher noise floor mainly due to the input voltage noise of the second OP-amp. The noise differences between the two solutions with the same bandwidth and gain can however often be neglected compared to the other noise sources in the system, as the laser and photodiode shot noise, laser phase nois, e and laser output noise [18]-[21]. If the same OP-amps are used in both the 
single and cascade case, the cascade solution has a higher theoretical maximum bandwidth for a given gain. Using only one amplifier will lower the power consumption and reduce the influence of external noise.

\section{REALIZATION}

The circuit has been assembled and tested in our fiber based system built around a one-section $10 \mathrm{~mW}$ DFB laser at $1510 \mathrm{~nm}$. We used the same component values as in Table I but a photodiode with $C_{d}=1.5 \mathrm{pF}$. Our local oscillator signal was achieved by utilizing the refractive index difference between the fiber core and air. Measuring the noise spectra was somewhat complicated since external noise effects dominated the spectra. To verify the transfer function we amplitude modulated our laser and compared the gain in the passband to the gain at lower and higher frequencies. The measured and simulated results showed decent agreement with deviations up to about $15 \%$. However, because of restrictions in the instruments we were not able to verify the lowest pole around $0.01 \mathrm{~Hz}$, as seen in Fig. 4. In our setup, and with our suggested amplifier circuit, with a transimpedance gain of just below $1 \mathrm{M} \Omega$ in the passband, the output voltage offset due to the dc current $I_{D}$ was just below $200 \mathrm{mV}$. As the maximum supply voltage of our OP-amp is $5.5 \mathrm{~V}$, a regular current-to-voltage converter with a transimpedance gain of only $56 \mathrm{k} \Omega$ saturated when tested in our lab system.

\section{CONCLUSION}

We have presented a single OP-amp solution that can combine minimal gain at dc with a high gain in the desired frequency region without the use of inductors. This makes it suitable for use in an optical self-mixing FMCW system. The circuit has been analyzed theoretically, simulated using SPICE and tested in our system. Unfortunately the simulated and theoretical noise results were difficult to verify practically. One drawback associated with adding $R_{4}$ and $C_{4}$ is that reverse biasing in most cases becomes necessary, and the resulting reverse bias will depend on the generated dc current. Compared to a cascade of two OP-amps, the theoretical noise performance of our solution is similar or slightly better. Using only one amplifier can also reduce the power consumption, the size of the circuit and the influence of external noise. The cascade solution does, however, have a slightly higher theoretical bandwidth for a given gain.

\section{REFERENCES}

[1] A. R. Slotwinski, F. E. Goodwin, and D. L. Simonson, "Utilizing AlGaAs laser diodes as a source for frequency modulated continuous wave (FMCW) coherent laser radar," in Proc. SPIE , Laser Diode Technology and Applications, vol. 1043, 1989, pp. 245-251.

[2] S. L. Vazquez, P. W. Goode, and A. R. Slotwinski, "Proportional proximity sensing for telerobots using coherent laser radar," SPIE, vol. 1829, pp. 25-35, 1992.

[3] A. Dieckmann and M.-C. Amann, "Frequency-modulated continuous-wave (FMCW) lidar with tunable twin-guide laser diod," SPIE, vol. 2249, pp. 22-30, 1994.

[4] R. L. Sebastian, R. B. Clark, D. L. Simonson, and A. R. Slotwinski, "Fiber optic coherent laser radar 3D vision system," SPIE, vol. 2348, pp. 266-274, 1995.

[5] C. Karlsson, D. Letalick, and I. Renhorn, Measurements of Noise and Signal-To-Noise Ratio for a Coherent Semiconductor Laser Radar, 1996.
[6] M. Millnert, C. Carlsson, D. Letalick, and I.Ingemar Renhorn, "Robust coherent laser radar design and signal processing for vibrometry," SPIE, vol. 2748, 1996.

[7] U. Haberland, V. Blazek, and J. H. Schmitt, "Optical coherent tomography with electrically tunable semiconductor laser using FMCW techniques," SPIE, vol. 2925, pp. 227-236, 1996.

[8] I. Koichi, W. Lu-Tang, and H. Ken-ichi, "Linearizing optical frequencysweep of a laser diode for FMCW reflectometry," J. Lighwave Technol., vol. 14, no. 2, pp. 173-178, 1996.

[9] P. Ojala et al., "Narrow-linewidth semiconductor lasers for coherent laser radar," in Proc. 9th Conf. Coherent Laser Radar, Linköping, Sweden, June 1997, pp. 70-73.

[10] C. Karlsson and D. Letalick, "Frequency modulation of a DFB laser diode for CLR applications," in Proc. 9th Conf. Coherent Laser Radar, Linköping, Sweden, June 1997, pp. 156-159.

[11] M. Nöding and M.-C. Amann, "Influence of nonlinear tuning characteristic of widely tunable laser diodes on FMCW-LIDAR performance," in Proc. EOS 14th Topical Meeting on Optoelectronic Distance/Displacement Measurements and Applications, Nantes, France, July 1997.

[12] P. Nerin et al., "Absolute distance and velocity measurements by FMCW technique and self-mixing interference inside a single mode Nd:YAG-LiTaO3 microchip laser," in Proc. EOS 14th Topical Meeting on Optoelectronic Distance/Displacement Measurements and Applications, Nantes, France, July 1997.

[13] C. J. Karlsson and F. A. Olsson, "Linearization of the frequency sweep of a frequency-modulated continuous-wave semiconductor laser radar and the resulting ranging performance," Appl. Opt., vol. 38, no. 15, pp. 3376-3386, 1999.

[14] U. Minoni, G. Scotti, and F. Docchio, "Wide-range distance meter based on frequency modulation of an Nd:YAG laser," Opt. Eng., vol. 35, no. 7, 1996.

[15] K. J.Kjell J. Gåsvik, Optical Metrology, 2nd ed. New York: Wiley, 1995, pp. 43-44.

[16] D. Nordin and K. Hyyppä, "Dividing optical power for maximum signal-to-noise ratio in a self mixing FMCW system," in EOS/SPIE Symp. Remote Sensing, Barcelona, Spain, Sept. 2000.

[17] J. Graeme, Photodiode Amplifiers, McGraw-Hill, New York, pp. $151-156,1996$

[18] V. Shalini and S. V. Wayne, "Phase noise considerations in coherent optical FMCW reflectometry," J. Lighwave Technol., vol. 11, no. 10, pp. 1694-1699, 1993.

[19] R. Passy, N. Gisin, J. P. von der Weid, and H. H. Gilgen, "Experimental and theoretical investigations of coherent OFDR with semiconductor laser sources," J. Lighwave Technol., vol. 12, no. 9, pp. 1622-1630, 1994.

[20] C. H. Henry, "Phase noise in semiconductor lasers," J. Lighwave Technol., vol. LT-4, no. 3, pp. 298-311, 1986.

[21] G. Economou, R. C. Youngquist, and D. E. N. Davies, "Limitations and noise in interferometric systems using frequency ramped single-mode diode lasers," J. Lighwave Technol., vol. LT-4, no. 11, pp. 1601-1608, 1986.

Daniel Nordin was born in Sweden in 1974. He received the M.S. degree in electrical engineering from the Luleå University of Technology, Luleå, Sweden, in 1999 . He is currently pursuing the Ph.D. degree at the EISLAB Division, Luleå University of Technology.

His main research interests include frequency modulation of diode lasers and optical ranging.

Kalevi Hyyppä received his M.S.E.E. degree from the Royal Institute of Technology in Stockholm, Sweden, in 1967 and the Ph.D. degree from the Lulea University of Technology, Luleå, Sweden, in 1993.

Over several years, he developed equipment for research on particle accelerators and rocket-borne instruments for plasma physics research in the ionosphere. After moving to the Luleå University of Technology in 1975, he developed optoelectronic measurement equipment for industrial use.

In 1991, he received the "Engineer of the Year" award in Sweden in recognition of a successful development of an optoelectronic navigation system for mobile robots. His current research interests are optoelectronics, mechatronics, and mobile robots. 